\title{
CORRECTIONS
}

\section{Could routine cardiotocography reduce long term cognitive impairment?}

Christoph Lees, one of the authors of this Analysis article by Charlotte Dyson and colleagues (BMJ 2011;342:d3120, doi:10. $1136 /$ bmj.d3120), is visiting professor at the Katholieke

Universiteit in Leuven (not the Kathalieke Universitiet as we misspelt it in the print version). Lees advised us of this affiliation after the online version of his article had been published.

Cite this as: $B M J 2011 ; 342: \mathrm{d} 3844$ 